\title{
Astaxanthin reduces type 2 diabetic-associated cognitive decline in rats via activation of PI3K/Akt and attenuation of oxidative stress
}

\author{
XIAOBIN $\mathrm{LI}^{1}$, ZHONGHUA QI ${ }^{2}$, LONGSHAN ZHAO ${ }^{3}$ and ZHAN YU ${ }^{4}$ \\ Departments of ${ }^{1}$ Emergency and ${ }^{2}$ Neurology, The First Affiliated Hospital of Dalian Medical University, Dalian, Liaoning 116011; \\ ${ }^{3}$ Department of Pharmacy, Shenyang Pharmaceutical University, Shenyang, Liaoning 110034; \\ ${ }^{4}$ Department of Pharmacy, The First Affiliated Hospital of Dalian Medical University, Dalian, Liaoning 116011, P.R. China
}

Received October 13, 2014; Accepted June 30, 2015

DOI: $10.3892 / \mathrm{mmr} .2015 .4615$

\begin{abstract}
Astaxanthin (AST) is an oxygenated derivative of carotenoid, which possesses a strong antioxidant activity. AST can effectively remove active oxygen from the body, and is thus considered to have an important role in disease prevention and treatment. The present study aimed to determine the effects of AST on type 2 diabetic-associated cognitive decline (DACD) in rats. Rats were intraperitoneally injected with streptozotocin (STZ), in order to establish a model of diabetes mellitus (DM). A total of 40 rats were randomly divided into five groups: The control group, the DM group, the AST (50 mg/kg) group, the AST (100 mg/kg) group, and the AST+LY294002 group (AST, $50 \mathrm{mg} / \mathrm{kg}$ and LY, $0.25 \mu \mathrm{g} / 100 \mathrm{~g}$ ). Following a 14-day treatment with AST, the body weight, blood glucose levels and cognitive function were determined. In addition, the protein expression levels of phosphatidylinositol 3-kinase (PI3K)/Akt, glutathione peroxidase and superoxide dismutase activity, glutathione and malondialdehyde content, and inducible nitric oxide synthase (iNOS), caspase-3 and caspase-9 activity were detected in the rats with DM. AST clearly augmented body weight and reduced blood glucose levels in rats with DM. Furthermore, treatment with AST significantly improved the cognitive function of rats with DM. Treatment with AST activated the PI3K/Akt pathway, and suppressed oxidative stress in the DM rats. In the cerebral cortex and hippocampus of the rats with DM, the activities of iNOS, caspase-3 and caspase-9 were markedly reduced. Furthermore, treatment with the Akt inhibitor LY294002 reduced the effectiveness of AST on DACD in rats. In conclusion, AST may reduce type 2 DACD in rats via activation of PI3K/Akt and attenuation of oxidative stress.
\end{abstract}

Correspondence to: Mr. Zhonghua Qi, Department of Neurology, The First Affiliated Hospital of Dalian Medical University, 222 Zhongshan Road, Dalian, Liaoning 116011, P.R. China E-mail: zhonghuaqihj@163.com

Key words: astaxanthin, type 2 diabetic-associated cognitive decline, phosphatidylinositol 3-kinase/Akt, inducible nitric oxide synthase

\section{Introduction}

Recent studies regarding type 2 diabetes mellitus (DM) have demonstrated that DM is an independent risk factor that can lead to diabetic-associated cognitive decline (DACD), and even dementia (1-3). However, the exact mechanism of type 2 DACD is currently unclear, and there are various factors, such as glucose metabolism, vascular structural function abnormalities, diabetic complications, and aging that may be involved in its pathogenesis. In addition, an overlap phenomenon exists between these various risk factors (4-6).

DACD dysfunction includes increased inducible nitric oxide synthase (iNOS)/nitric oxide (NO) expression, oxidative stress and the production of various cytokines (7). iNOS is a key enzyme that regulates the generation of endothelial-derived NO via the phosphoinositide 3-kinase (PI3K)/Akt signaling pathway. The PI3K/Akt signaling pathway has an important role in the regulation of cellular metabolism, growth, migration and proliferation. Zhu et al (8) previously demonstrated that ganoderma atrum polysaccharide was able to increase the aortic relaxation of diabetic rats via activation of the PI3K/Akt signal pathway. In addition, Jia et al (9) reported that fish oil augmented learning impairments of diabetic rats through upregulating the PI3K/AKT pathway (9).

The process of oxidative stress is inseparable from protein glycosylation. The high blood sugar levels associated with DM lead to the increased formation of advanced glycation end-products (AGEs), which accumulate in the blood vessel walls and interfere with endogenous NO synthesis and vasodilatation $(10,11)$. Low-density lipoprotein (LDL) oxidative modification weakens the recognition by the receptor (12), reducing LDL clearance and resulting in elevated LDL levels (13). Oxidative stress has an important role in the activation and acceleration of arteriosclerosis. Furthermore, the chemical structure, and cell and tissue effects of AGEs accelerate aging-related alterations; previous studies have demonstrated that oxidative stress and the formation of AGEs may lead to DACD (14-16).

Astaxanthin (AST) is an oxygen-containing derivative of carotenoids, which can effectively quench activated oxygen, and has high nutritional and medicinal value (17). In the 1930s, AST was separated from shrimp and crab shells; however, the physiological function of AST did not gather attention until the 
1980s (18). Since then, numerous studies have demonstrated that AST is able to inhibit tumorigenesis, enhance immune function, and prevent a wide range of physiological conditions, such as cardiovascular disease (19). AST is therefore considered to possess broad application prospects in animal studies and clinical trials (20). A recent study demonstrated that AST could attenuate diabetes through its effects on oxidative stress and inflammation (21). However, despite the promising evidence regarding the effects of AST on diabetes, little is currently known regarding its effects on the amelioration of DACD in diabetic rats. Therefore, the present study aimed to investigate whether AST was able to attenuate pathological changes on DACD, and to explore the underlying molecular signaling pathway of this beneficial effect.

\section{Materials and methods}

Drugs and chemicals. AST (purity $\geq 97 \%$; Fig. 1) and streptozotocin (STZ) were purchased from Sigma-Aldrich (St. Louis, MO, USA). Glucose radioimmunoassay kit was purchased from Nanjing Jiancheng Bioengineering Institute (Nanjing, China). Bicinchoninic acid (BCA) protein assay kit, the glutathione peroxidase (GPx) and superoxide dismutase (SOD) activity assay kits, and the glutathione (GSH) and malondialdehyde (MDA) content assay kits were purchased from BestBio (Shanghai, China). An iNOS assay kit (cat. no. E-CLR0358c; Wuhan Elabscience Biological Technology Co., Ltd., Wuhan, China), and caspase-3 and caspase-9 activity assay kits (cat. no. E-EL-R0160c; Wuhan Elabscience Biological Technology Co., Ltd; cat. no. C1158, Beyotime Institute of Biotechnology (Haimen, China) were also purchased.

Experimental animals. The present study was conducted in accordance with the Principles of Laboratory Animal Care, and the Guide for the Care and Use of Laboratory Animals at Dalian Medical University (Dalian, China). Sprague-Dawley (SD) rats ( $n=40$; age, 8 weeks; weight, 200-230 g) were purchased from the Comparative Medicine Center, Dalian Medical University and approved by the ethics committee of The First Affiliated Hospital of Dalian Medical University. All of the rats were allowed to acclimatize in an animal room (temperature, 22-25 ${ }^{\circ} \mathrm{C}$; humidity, 55-60\%; $12 \mathrm{~h}$ light/dark cycle), and were provided access to chow and water ad libitum.

Establishment of the model and measurement of DM. After 1 week acclimatization, each rat was injected intraperitoneally (i.p.) with $65 \mathrm{mg} / \mathrm{kg} \mathrm{STZ} \mathrm{(pH} \mathrm{4.4),} \mathrm{in} \mathrm{order} \mathrm{to} \mathrm{induce} \mathrm{DM.} \mathrm{A}$ total of $48 \mathrm{~h}$ after STZ injection, fasting blood glucose levels were determined using a radioimmunoassay kit (Nanjing Jiancheng Bioengineering Institute). Rats with fasting blood glucose levels $>250 \mathrm{mg} / \mathrm{dl}$ were considered diabetic, and were used for further experimentation.

Experimental groups. A total of 40 rats were randomly divided into five groups: (i) The control (Con) group $(n=8)$, which consisted of rats treated with i.p. isotonic $\mathrm{NaCl}$; (ii) the DM group ( $n=8)$, which consisted of diabetic rats treated with i.p. saline; (iii) the AST (50) group $(n=8)$, which consisted of diabetic rats treated with i.p. $50 \mathrm{mg} / \mathrm{kg}$ AST; (iv) the AST (100) group $(n=8)$, which consisted of diabetic rats treated with

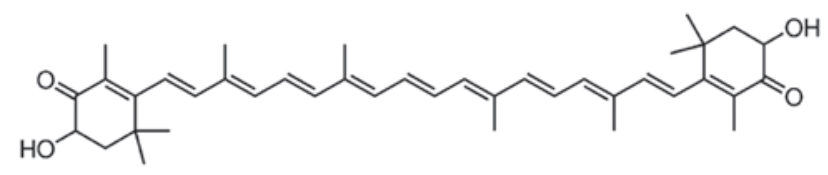

Figure 1. Chemical structure of astaxanthin.

i.p.100 mg/kg AST; and (v) the AST+LY294002 (AST+LY; Sigma-Aldrich) group $(n=8)$, which consisted of diabetic rats treated with i.p. $50 \mathrm{mg} / \mathrm{kg}$ AST and $0.25 \mu \mathrm{g} / 100 \mathrm{~g} \mathrm{LY} 294002$.

Morris water maze test. Following a 14-day treatment with AST, cognitive function was evaluated using the Morris water maze test, as described previously (22). Each rat was trained five times a day at 20 min intervals. The test was performed blindly every day for 5 days. Swimming was video tracked (ANY-maze; Stoelting Co., Wood Dale, IL, USA), and latency, path length, swim speed, and cumulative distance from the platform was recorded. Mean swim latency for all of the rats was evaluated on each day. After a training trial, the mean time spent in the correct quadrant containing the platform, and the mean number of times the mice crossed the former platform position during $60 \mathrm{sec}$ was estimated.

Western blotting of phosphorylated (p)-Akt and Akt. To determine protein concentrations, blood was obtained from every rat by retro-orbital blood collection under anesthetization (pentobarbital sodium, $30 \mathrm{mg} / \mathrm{kg}$, P11011; Merck KGaA, Darmstadt, Germany) and $50 \mathrm{mg}$ brain tissue from the cerebral cortex/hippocampus was removed, which was incubated with $100 \mu \mathrm{l}$ tissue lysis buffer on ice for $30 \mathrm{~min}$ and centrifuged at $20,000 \mathrm{x}$ g for $15 \mathrm{~min}$ at $4^{\circ} \mathrm{C}$. Protein concentration was then determined using the BCA protein assay kit. For each sample, $50 \mu \mathrm{g}$ of protein was separated by SDS-PAGE (Beyotime Institute of Biotechnology) and transferred to a nitrocellulose membrane (Bio-Rad Laboratories, Inc., Hercules, CA, USA). The membrane was then blocked with 5\% non-fat milk in PBS for $3 \mathrm{~h}$, and washed in Tris-buffered saline containing Tween-20 (Beyotime Institute of Biotechnology) overnight at $4^{\circ} \mathrm{C}$. Protein bands were detected using a BCIP-NBT kit (Promega Corporation, Madison, WI, USA). The antibodies used were as follows: Monoclonal rabbit anti-mouse anti-p-Akt (1:800 dilution; cat. no. sc-293094; Santa Cruz Biotechnology, Inc., Dallas, TX, USA), monoclonal rabbit anti-mouse anti-Akt (1:1,000 dilution; cat. no. sc-7126; Santa Cruz Biotechnology, Inc.) and anti- $\beta$-actin (1:500 dilution; Wuhan Boster Biological Technology, Ltd., Wuhan, China) at $4^{\circ} \mathrm{C}$ overnight. After incubation with the primary antibodies, the membrane was incubated with horseradish peroxidase-conjugated anti-mouse IgG (ZF-0312; 1:5,000; Zhongshan Golden Bridge Biotechnology Co., Ltd., Beijing, China) for $1 \mathrm{~h}$ at room temperature, followed by incubation with ECL Super Signal ${ }^{\circledR}$ West Pico Chemiluminescent Substrate (Pierce Biotechnology, Inc., Rockford, IL, USA).

Measurement of GPx, GSH, SOD and MDA activities. Approximately 5-10 mg brain tissue was added to $100 \mu \mathrm{l}$ tissue lysis buffer (Beyotime Institute of Biotechnology). 

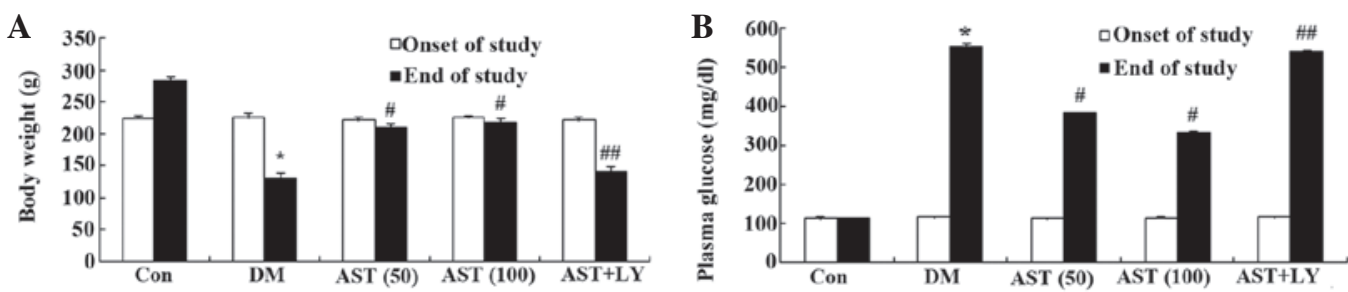

Figure 2. Effects of AST on (A) body weight and (B) blood glucose levels in rats. " $\mathrm{P}<0.01$, compared with the Con group; $\mathrm{P}<0.01$, compared with the DM group; ${ }^{\# P} \mathrm{P}>0.05$, compared with the DM group. AST, astaxanthin; Con, control group; DM, diabetes group; AST (50), AST (50 mg/kg)-treated group; AST (100), AST (100 mg/kg)-treated group; AST+LY, AST (50 mg/kg) and LY294002 (0.25 $\mu \mathrm{g} / 100 \mathrm{~g})$-treated group.

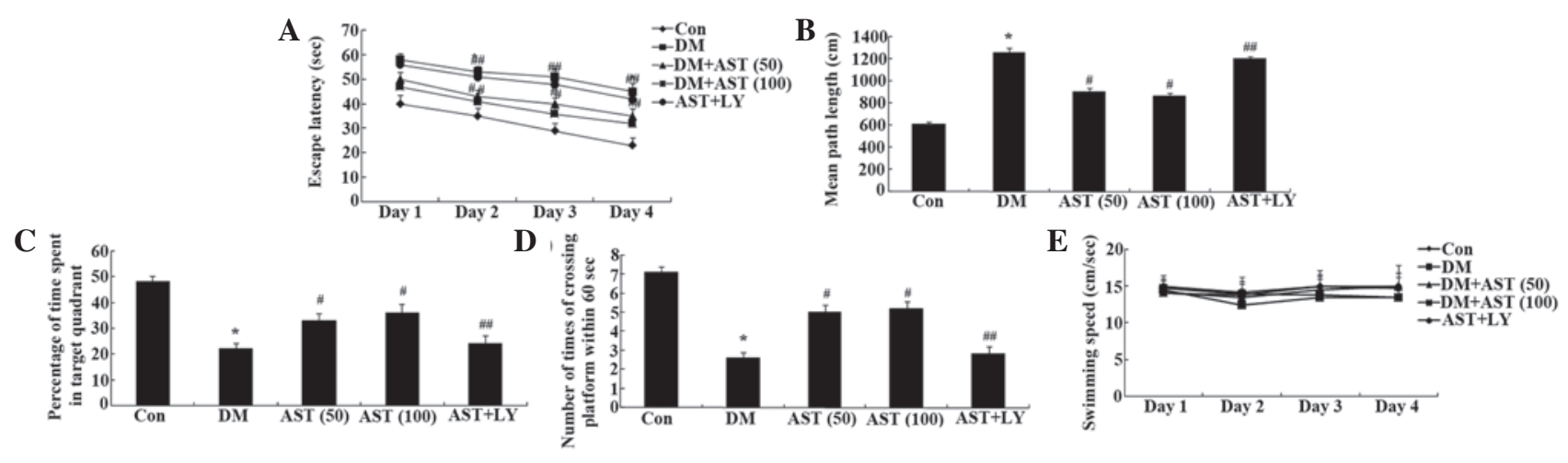

Figure 3. Effects of AST on diabetes-induced cognitive deficit. Effects of AST on the (A) escape latency, (B) mean path length, (C) mean percentage of time spent in the target quadrant, (D) the number of times the platform was crossed, and (E) swimming speed in rats. "P<0.01, compared with the Con group; ${ }^{\#} \mathrm{P}<0.01$, compared with the DM group; ${ }^{\# /} \mathrm{P}>0.05$, compared with the DM group. AST, astaxanthin; Con, control group; DM, diabetes group; AST (50), AST $(50 \mathrm{mg} / \mathrm{kg})$-treated group; AST (100), AST (100 mg/kg)-treated group; AST+LY, AST (50 mg/kg) and LY294002 (0.25 $\mu \mathrm{g} / 100 \mathrm{~g})$-treated group.

The tissue pellet was homogenized on ice, and the mixture was incubated for 5-10 $\mathrm{min}$. The supernatant was obtained following centrifugation at 16,000-20,000 x g for $15 \mathrm{~min}$ at $4^{\circ} \mathrm{C}$. The activities of GPx, GSH, SOD and MDA were detected using kits, according to the manufacturer's instructions. Serum GPx activity was detected by measuring the absorbance at $340 \mathrm{~nm}$ using a plate reader (Wallac VICTOR 1420; PerkinElmer, Inc., Waltham, MA, USA). The levels of GPx and SOD were expressed in $\mathrm{U} / \mathrm{mg}$. The levels of serum GSH and MDA levels were expressed in $\mu \mathrm{g} / \mathrm{g}$ and $\mathrm{nmol} / \mathrm{mg}$, respectively.

Measurement of iNOS activity. Approximately 5-10 mg brain tissue was added to $100 \mu \mathrm{l}$ tissue lysis buffer. The tissue pellet was homogenized on ice, and the mixture was incubated for 5-10 min. The supernatant was obtained following centrifugation at 16,000-20,000 x g for $15 \mathrm{~min}$ at $4^{\circ} \mathrm{C}$. iNOS activity was measured using an iNOS assay kit in accordance with the manufacturer's instructions. The absorbance was measured at a wavelength of $530 \mathrm{~nm}$ to determine iNOS activity.

Measurement of caspase-3 and caspase-9 activity. Approximately 5-10 mg brain tissue was added to $100 \mu \mathrm{l}$ tissue lysis buffer. The tissue pellet was homogenized on ice, and the mixture was incubated for 5-10 min. The supernatant was obtained following centrifugation at 16,000-20,000 x g for $15 \mathrm{~min}$ at $4^{\circ} \mathrm{C}$. Caspase- 3 and caspase- 9 activity was measured using activity assay kits, according to the manufacturer's instructions; the samples were incubated at $37^{\circ} \mathrm{C}$ for $2 \mathrm{~h}$. Absorbance was measured at a wavelength of $405 \mathrm{~nm}$ to determine caspase- 3 and caspase- 9 activity.

Statistical analysis. Data are expressed as the mean \pm standard deviation. Statistical significance was analyzed using one-way analysis of variance followed by Dunnett's test. SPSS 17.0 software (SPSS, Inc., Chicago, IL, USA) was used for statistical analysis. $\mathrm{P}<0.05$ was considered to indicate a statistically significant difference.

\section{Results}

Effects of AST on body weight and blood glucose levels. The present study determined whether AST could influence the body weight and blood glucose levels of diabetic rats. The body weight of the DM group was successfully reduced, as compared with the control group (Fig. 2A). In addition, the blood glucose levels of the DM group were notably increased, as compared with the control group (Fig. 2B). Following a 14-day treatment with AST, the body weight and blood glucose levels were markedly augmented and reduced, as compared with the control group (Fig. 2A and B). However, the body weight and blood glucose levels of the AST+LY group were similar to the DM group (Fig. 2A and B).

Effects of AST on cognitive function. The escape latency of the DM rats was markedly increased, as compared with the control rats (Fig. 3A). Following a 14-day treatment with AST, the escape latency of the DM rats was significantly reduced, as compared with the DM group (Fig. 3A). 
A

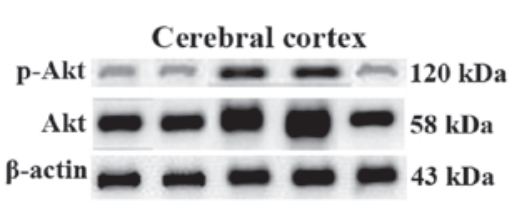

C

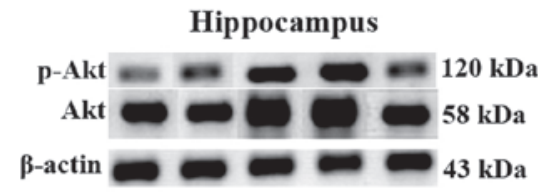

D
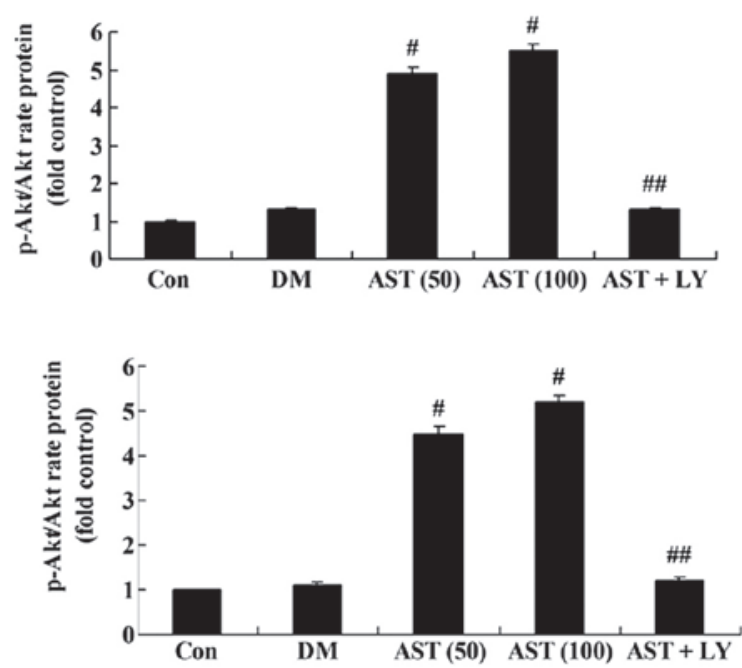

Figure 4. Effects of AST on diabetes-induced changes in p-Akt/Akt expression in the cerebral cortex and hippocampus Representative western blot analyses and statistical analysis of the protein expression levels of p-Akt and Akt p in the (A and B) cerebral cortex and (C and D) hippocampus. ${ }^{*} \mathrm{P}<0.01$, compared with the DM group; ${ }^{\# /} \mathrm{P}>0.05$, compared with the DM group. AST, astaxanthin; Con, control group; DM, diabetes group; AST (50), AST (50 mg/kg)-treated group; AST (100), AST (100 mg/kg)-treated group; AST+LY, AST (50 mg/kg) and LY294002 (0.25 $\mu \mathrm{g} / 100 \mathrm{~g})$-treated group.

A

C
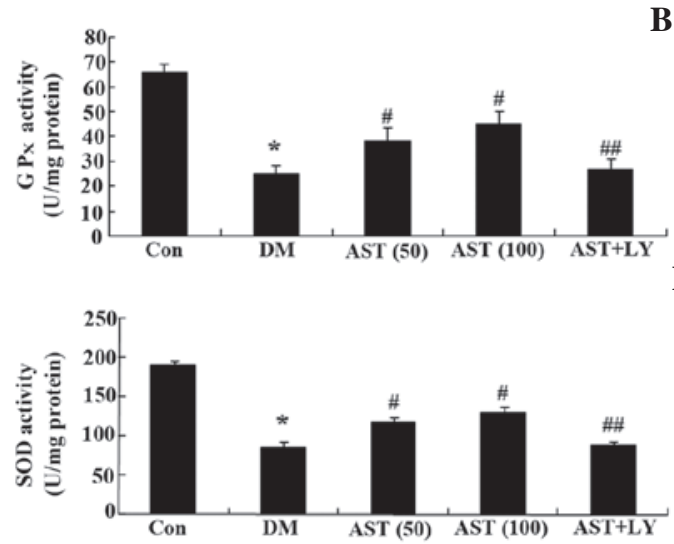

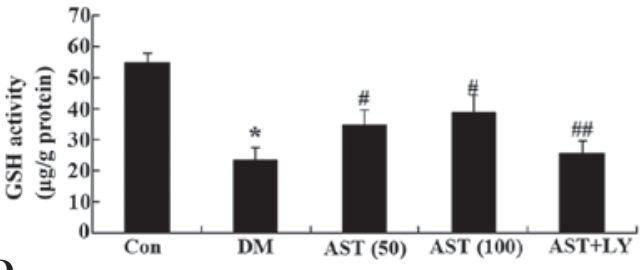

D

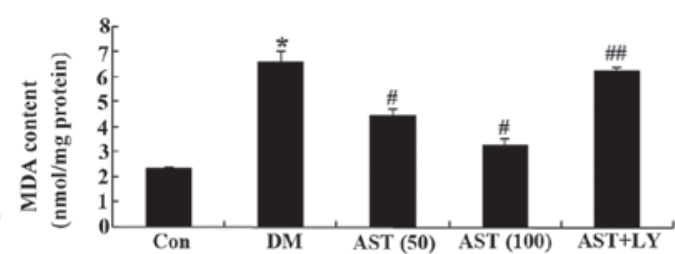

Figure 5. Effects of AST on diabetes-induced changes in GPx, SOD, GSH and MDA levels. Effects of AST on the levels of (A) GPx, (B) GSH and (C) SOD, and (D) the MDA content in rats. "P $<0.01$, compared with the Con group; ${ }^{\#} \mathrm{P}<0.01$, compared with the DM group; ${ }^{\# \prime} \mathrm{P}>0.05$, compared with the DM group. AST, astaxanthin; GSH, glutathione; GPx, glutathione peroxidase; SOD, superoxide dismutase; MDA, malondialdehyde; Con, control group; DM, diabetes group; AST (50), AST (50 mg/kg)-treated group; AST (100), AST (100 mg/kg)-treated group; AST+LY, AST (50 mg/kg) and LY294002 (0.25 $\mu \mathrm{g} / 100 \mathrm{~g})$-treated group.

Mean path length was markedly increased in the DM rats, as compared with the control rats, whereas the mean path length of the AST-treated rats was significantly decreased, as compared with the DM rats (Fig. 3B). Furthermore, there was a marked reduction in the time spent in the target quadrant for the DM rats, whereas the time spent in the target quadrant for the AST rats was significantly increased, as compared with the DM rats (Fig. 3C). Notably, the number of times the rats crossed the former platform location was also decreased for the DM rats, as compared with the control group, whereas the number of times the rats crossed the former platform location was significantly increased for the AST rats, as compared with the DM rats (Fig. 3D). There was no significant difference in swimming speed between the various groups (Fig. 3E). Notably, the cognitive function of the AST+LY group was similar to that of the DM group (Fig. 3).

Effects of AST on diabetes-induced changes in p-Akt and Akt expression. To assess the impact of AST on the expression

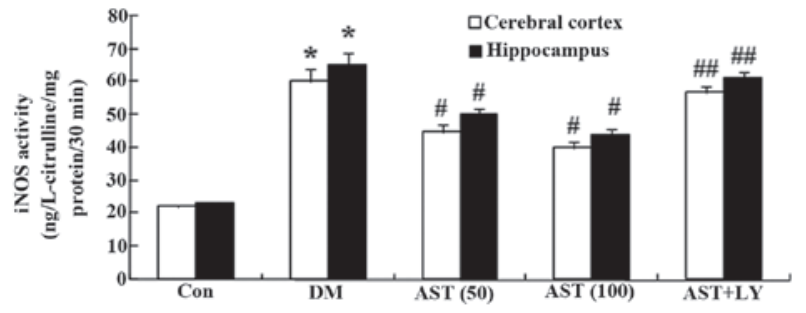

Figure 6. Effects of AST on iNOS activity. Effects of AST on diabetes-induced alterations in iNOS activity. "P $<0.01$, compared with the Con group; ${ }^{\text {}} \mathrm{P}<0.01$, compared with the DM group; ${ }^{\# \prime} \mathrm{P}>0.05$, compared with the DM group. AST, astaxanthin; iNOS, inducible nitric oxide synthase; Con, control group; DM, diabetes group; AST (50), AST (50 mg/kg)-treated group; AST (100), AST $(100 \mathrm{mg} / \mathrm{kg})$-treated group; AST+LY, AST $(50 \mathrm{mg} / \mathrm{kg})$ and LY294002 $(0.25 \mu \mathrm{g} / 100 \mathrm{~g})$-treated group.

levels of Akt proteins in the brain cells of rats, the expression levels of Akt proteins were detected in the cerebral cortex and hippocampus. Treatment with AST (50 and $100 \mathrm{mg} / \mathrm{kg}$ ) 

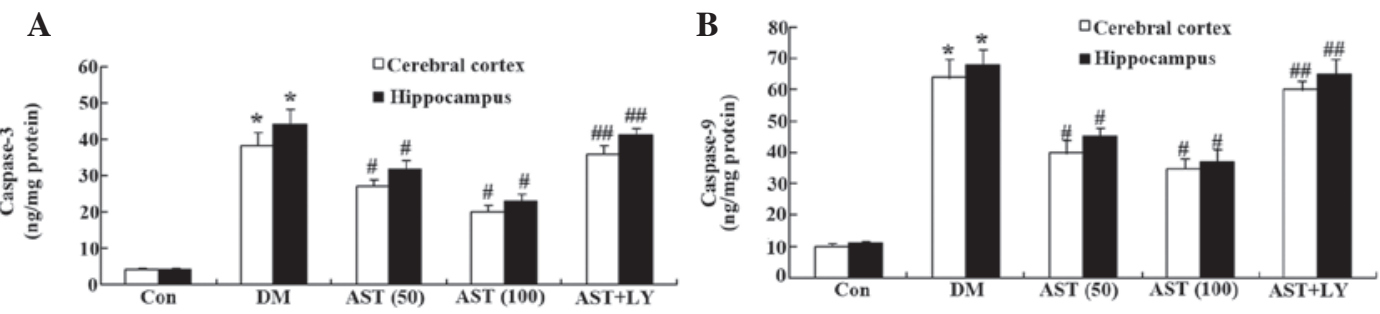

Figure 7. Effects of AST on caspase-3 and caspase-9 activity. Effects of AST on (A) caspase- 3 and (B) caspase- 9 activity in rats. ${ }^{*} \mathrm{P}<0.01$, compared with the Con group; ${ }^{\#} \mathrm{P}<0.01$, compared with the DM group; ${ }^{\# \#} \mathrm{P}>0.05$, compared with the DM group. AST, astaxanthin; Con, control group; DM, diabetes group; AST (50), AST $(50 \mathrm{mg} / \mathrm{kg})$-treated group; AST (100), AST (100 mg/kg)-treated group; AST+LY, AST (50 mg/kg) and LY294002 $(0.25 \mu \mathrm{g} / 100 \mathrm{~g})$-treated group.

significantly activated the expression of p-Akt and Akt in the cerebral cortex and hippocampus of diabetic rats, as compared with the DM group (Fig. 4). The protein expression levels of p-Akt and Akt in the AST+LY group were similar to in the DM group (Fig. 4).

Effects of AST on diabetes-induced changes in GPx, GSH, $S O D$ and MDA. To clarify the anti-oxidative effects of AST on diabetic rats, the levels of GPx, GSH, SOD and MDA were detected. Following a 14-day treatment, the levels of GPx, GSH and SOD in the cerebral cortex and hippocampus of the DM group were markedly decreased, as compared with the control group (Fig. 5A-C). However, the levels of GPx, GSH and SOD in the cerebral cortex and hippocampus of the diabetic rats were markedly increased following a 14-day treatment with AST, as compared with the DM group (Fig. 5A-C). Furthermore, the GPx, GSH and SOD levels in the AST+LY group were similar to those in the DM group (Fig. 5A-C).

Simultaneously, the MDA content in the cerebral cortex and hippocampus of the rats with DM was significantly increased, as compared with the control group (Fig. 5D). However, the MDA content in the cerebral cortex and hippocampus of the DM rats was significantly decreased following a 14-day treatment with AST, as compared with the DM group (Fig. 5D). Furthermore, the MDA content of the AST+LY group was similar to that in the DM group (Fig. 5D).

Effects of AST on iNOS activity. To investigate whether AST exerted protective effects via reducing NOS production, the activity of iNOS was detected. iNOS activity in the cerebral cortex and hippocampus of the DM group was increased, as compared with the control group (Fig. 6). However, iNOS activity in the cerebral cortex and hippocampus of diabetic rats was decreased following a 14-day treatment with AST, as compared with the DM group (Fig. 6). Furthermore, the iNOS activity of the AST+LY group was similar to the DM group (Fig. 6).

Effects of AST on caspase-3 and caspase-9 activity. To evaluate the effects of AST on cellular apoptosis in diabetic rats, caspase- 3 and caspase- 9 activity was measured in the cerebral cortex and hippocampus. Caspase- 3 and caspase- 9 activity in the cerebral cortex and hippocampus of the DM group was markedly increased, as compared with the control group (Fig. 7A and B). However, treatment with AST (50 and
$100 \mathrm{mg} / \mathrm{kg}$ ) significantly reduced caspase- 3 and caspase- 9 activity in the cerebral cortex and hippocampus of diabetic rats following a 14-day treatment, as compared with the DM group (Fig. 7A and B). Furthermore, caspase-3 and caspase-9 activity was similar in the AST+LY group, as compared with the DM group (Fig. 7A and B).

\section{Discussion}

Type 2 (T2) DM is a major cause of morbidity and mortality worldwide, and the prevalence of T2DM is increasing (23). The greater proportion of this is in Asia, Africa and South America. The biological mechanisms linking T2DM with impaired DACD remain unclear. Individuals with T2DM are at an enhanced risk for stroke, which is a risk factor for cognitive decline, and may also contribute to microvascular alterations and cerebral ischemia $(24,25)$. The present study demonstrated that AST was able to markedly augment body weight and reduce the blood glucose levels of DM rats. Naito et al (26) previously reported that AST reduced blood glucose levels and increased body weight in a rodent model of T2DM. However, the effects of AST on the body weight and blood glucose levels of rats with DM were reversed by LY294002. Furthermore, the cognitive function of DM rats was improved following treatment with AST, whereas treatment with the Akt inhibitor LY294002 reduced the cognitive function of DM rats.

A recent study demonstrated that activation of the PI3K/Akt pathway serves as a pro-survival signal in the protection of nerve cells, and has an important role in hypoxic-ischemic neuronal damage (27). The PI3K/Akt pathway controls cell survival by regulating apoptosis and autophagy in the nervous system (28). Activation of PI3K and Akt can promote endothelial cell survival, reduce nerve damage, reduce inflammatory cell death, and block neuronal damage $(29,30)$. Insulin-like growth factor, hypothermia and ischemic preconditioning also have a protective role in the brain via the PI3K/Akt signaling pathway (31). In the present study, in the cerebral cortex and hippocampus, the protein expression levels of p-Akt and Akt were significantly upregulated by treatment with AST in the rats with DM. However, treatment with AST $(50 \mathrm{mg} / \mathrm{kg})$ with LY294002 $(0.25 \mathrm{mg} / \mathrm{kg})$ was able to weaken the expression levels of p-Akt and Akt in rats with DM. Li et al (32) reported that AST decreased oxidative stress of ARPE-19 cells via activation of PI3K/Akt. In addition, treatment with AST increased cell apoptosis in a hamster model of oral cancer via inactivation of extracellular signal-regulated kinases/mitogen-activated protein kinases and PI3K/Akt pathways (33). Furthermore, Zhang et al (34) reported that the 
PI3K/Akt inhibitor, LY294002, was able to partially reverse the neuroprotection of AST in the early period after subarachnoid hemorrhage, by downregulating AST-induced activation of Akt.

Numerous studies have focused on the association between oxidative stress and DACD. Free radicals are highly reactive, and can exert strong oxidative effects that damage biological macromolecules and numerous cellular components, and have significant toxic effects on nerve cells that can lead to lipofuscin deposition, increasing age spots, and vacuolar degeneration (35). In the present study, AST markedly increased GPx, GSH and SOD levels, and decreased MDA content in the cerebral cortex and hippocampus of diabetic rats. Notably, LY294002 effectively reduced the protective effects of AST on the oxidative stress of rats with DM. Wu et al (36) demonstrated that AST was able to alleviate brain aging in rats through repairing the activities of GPx and SOD, increasing GSH content, and reducing MDA content. Augusti et al (37) indicated that AST may reduce oxidative stress in rabbits with atherosclerosis.

The present study demonstrated that in the cerebral cortex and hippocampus, iNOS activity was decreased following treatment with AST; however, the effects of AST on iNOS activity in DM rats were reduced by LY294002. Choi et al (38) previously suggested that AST was able to inhibit the production of inflammatory mediators by blocking iNOS activation. In addition, AST may inhibit NO production by suppressing nuclear factor- $\kappa$ B activation (39). Simultaneously, in the present study AST protected brain cells and reduced the cell apoptosis of DM rats by suppressing caspase- 3 and caspase- 9 activity. Song et al (40) demonstrated that AST inhibited apoptosis of alveolar epithelial cells via inhibition of cytochrome $c$ release, and caspase- 9 and caspase- 3 activation (40). In addition, AST has been shown to protect against 1-methyl-4-phenyl-1,2,3,6 -tetrahydropyridine/1-methyl-4-phenylpyridinium-induced mitochondrial dysfunction via inhibition of the activation of caspase-3 (22).

In conclusion, the present study demonstrated that treatment with AST may reduce type 2 DACD in rats via activation of PI3K/Akt and downregulation of the oxidative stress pathway. These results suggest that AST may protect against cognitive decline in rats with T2DM.

\section{References}

1. Iwanami J, Mogi M, Tsukuda K, Jing F, Ohshima K, Wang XL, Nakaoka H, Kan-no H, Chisaka T, Bai HY, et al: Possible synergistic effect of direct angiotensin II type 2 receptor stimulation by compound 21 with memantine on prevention of cognitive decline in type 2 diabetic mice. Eur J Pharmacol 724: 9-15, 2014.

2. Hishikawa N, Yamashita T, Deguchi K, Wada J, Shikata K, Makino $\mathrm{H}$ and Abe $\mathrm{K}$ : Cognitive and affective functions in diabetic patients associated with diabetes-related factors, white matter abnormality and aging. Eur J Neurol 22: 313-321, 2015.

3. Wang YB, Wang S, Bai R, Du JL, Xing Q, Ba Y, Yang Y, Zhang XY, Shi $\mathrm{CH}$ and Yao JJ: Efficacy of switching from premixed insulin to insulin glargine regimen in Type 2 diabetes mellitus patients with different islet functions. Mol Med Rep 10: 1096-1102, 2014.

4. Lian G, Yue X, Xianxiang Z, Yong L, Weijuan L and Bing C: Insulinization: A promising strategy for the treatment of type 2 diabetes mellitus. Exp Ther Med 6: 1300-1306, 2013.

5. Mossello E, Ballini E, Boncinelli M, Monami M, Lonetto G, Mello AM, Tarantini F, Baldasseroni S, Mannucci E and Marchionni N: Glucagon-like peptide-1, diabetes, and cognitive decline: Possible pathophysiological links and therapeutic opportunities. Exp Diabetes Res 2011: 281674, 2011.
6. Marder TJ, Flores VL, Bolo NR, Hoogenboom WS Simonson DC, Jacobson AM, Foote SE, Shenton ME, Sperling RA and Musen G: Task-induced brain activity patterns in type 2 diabetes: A potential biomarker for cognitive decline. Diabetes 63: 3112-3119, 2014

7. Liu B, Kuang L and Liu J: Bariatric surgery relieves type 2 diabetes and modulates inflammatory factors and coronary endothelium eNOS/iNOS expression in $\mathrm{db} / \mathrm{db}$ mice. Can J Physiol Pharmacol 92: 70-77, 2014

8. Zhu KX, Nie SP, Li C, Gong D and Xie MY: Ganoderma atrum polysaccharide improves aortic relaxation in diabetic rats via PI3K/Akt pathway. Carbohydr Polym 103: 520-527, 2014.

9. Jia D, Heng LJ, Yang RH and Gao GD: Fish oil improves learning impairments of diabetic rats by blocking PI3K/AKT/nuclear factor- $\kappa \mathrm{B}$-mediated inflammatory pathways. Neuroscience 258: 228-237, 2014.

10. Lin X, Chen X, Ye J, Li Q, Zhou J, Wu X, Huang Y, Li X, Shi Y, $\mathrm{Li} \mathrm{S}, \mathrm{Li} \mathrm{L}$ and Cai H: Association between endogenous secretory receptor for advanced glycation-end products (esRAGE) and carotid intima-media thickness in type 2 diabetes. Exp Clin Endocrinol Diabetes 122: 277-280, 2014.

11. Si X, Li P, Zhang Y, Zhang Y, Lv W and Qi D: Renoprotective effects of olmesartan medoxomil on diabetic nephropathy in streptozotocin-induced diabetes in rats. Biomed Rep 2: 24-28, 2014.

12. Pietzsch J, Laube M, Bechmann N, Pietzsch FJ and Kniess T: Protective effects of 2,3-diaryl-substituted indole-based cyclooxygenase- 2 inhibitors on oxidative modification of human low density lipoproteins in vitro. Clin Hemorheol Microcirc Dec 29, 2014. [Epub ahead of print].

13. Price TO, Eranki V, Banks WA, Ercal N and Shah GN: Topiramate treatment protects blood-brain barrier pericytes from hyperglycemia-induced oxidative damage in diabetic mice. Endocrinology 153: 362-372, 2012.

14. Liu Y, Tian X, Gou L, Sun L, Ling X and Yin X: Luteolin attenuates diabetes-associated cognitive decline in rats. Brain Res Bull 94: 23-29, 2013.

15. Stinghen AE, Massy ZA, Vlassara H, Striker GE and Boullier A: Uremic Toxicity of Advanced Glycation End Products in CKD. J Am Soc Nephrol2015.

16. Jiao L, Stolzenberg-Solomon R, Zimmerman TP, Duan Z, Chen L, Kahle L, Risch A, Subar AF, Cross AJ, Hollenbeck A, et al: Dietary consumption of advanced glycation end products and pancreatic cancer in the prospective NIH-AARP Diet and Health Study. Am J Clin Nutr 101: 126-134, 2015.

17. Chang CS, Chang CL and Lai GH: Reactive oxygen species scavenging activities in a chemiluminescence model and neuroprotection in rat pheochromocytoma cells by astaxanthin, beta-carotene, and canthaxanthin. Kaohsiung J Med Sci 29: 412-421, 2013.

18. Visser H, van Ooyen AJ and Verdoes JC: Metabolic engineering of the astaxanthin-biosynthetic pathway of Xanthophyllomyces dendrorhous. FEMS Yeast Res 4: 221-231, 2003.

19. Wu H, Niu H, Shao A, Wu C, Dixon BJ, Zhang J, Yang S and Wang Y: Astaxanthin as a Potential Neuroprotective Agent for Neurological Diseases. Mar Drugs 13: 5750-5766, 2015.

20. Rao AR, Sindhuja HN, Dharmesh SM, Sankar KU, Sarada R and Ravishankar GA: Effective inhibition of skin cancer, tyrosinase, and antioxidative properties by astaxanthin and astaxanthin esters from the green alga Haematococcus pluvialis. J Agric Food Chem 61: 3842-3851, 2013.

21. Chan KC, Pen PJ and Yin MC: Anticoagulatory and antiinflammatory effects of astaxanthin in diabetic rats. J Food Sci 77: H76-H80, 2012.

22. Lee DH, Kim CS and Lee YJ: Astaxanthin protects against MPTP/MPP ${ }^{+}$-induced mitochondrial dysfunction and ROS production in vivo and in vitro. Food Chem Toxicol 49: 271-280, 2011.

23. Sun K, Chang X, Yin L, Li J, Zhou T, Zhang C and Chen X: Expression and DNA methylation status of microRNA-375 in patients with type 2 diabetes mellitus. Mol Med Rep 9: 967-972, 2014.

24. Petty MA and Lo EH: Junctional complexes of the blood-brain barrier: Permeability changes in neuroinflammation 68: 311-323, 2002.

25. Biessels GJ, Deary IJ and Ryan CM: Cognition and diabetes: A lifespan perspective. Lancet Neurol 7: 184-190, 2008.

26. Naito Y, Uchiyama K, Aoi W, Hasegawa G, Nakamura N, Yoshida N, Maoka T, Takahashi J and Yoshikawa T: Prevention of diabetic nephropathy by treatment with astaxanthin in diabetic $\mathrm{db} / \mathrm{db}$ mice. Biofactors 20: 49-59, 2004. 
27. Bulhak AA, Jung C, Ostenson CG, Lundberg JO, Sjöquist PO and Pernow J: PPAR-alpha activation protects the type 2 diabetic myocardium against ischemia-reperfusion injury: Involvement of the PI3-Kinase/Akt and NO pathway. Am J Physiol Heart Circ Physiol 296: H719-H727, 2009.

28. You JJ, Yang CH, Yang CM and Chen MS: Cyr61 induces the expression of monocyte chemoattractant protein-1 via the integrin $\alpha v \beta 3$, FAK, PI3K/Akt and NF- $\kappa \mathrm{B}$ pathways in retinal vascular endothelial cells. Cell Signal 26: 133-140, 2014.

29. Cho YR, Lim JH, Kim MY, Kim TW, Hong BY, Kim YS, Chang YS, Kim HW and Park CW: Therapeutic effects of fenofibrate on diabetic peripheral neuropathy by improving endothelial and neural survival in $\mathrm{db} / \mathrm{db}$ mice. PLoS One 9: e83204, 2014.

30. Malemud CJ: The PI3K/Akt/PTEN/mTOR pathway: a fruitful target for inducing cell death in rheumatoid arthritis? Future Med Chem 7: 1137-1147, 2015

31. Wang T, Mao X, Li H, Qiao S, Xu A, Wang J, Lei S, Liu Z, Ng KF, Wong GT, et al: N-Acetylcysteine and allopurinol up-regulated the Jak/STAT3 and PI3K/Akt pathways via adiponectin and attenuated myocardial postischemic injury in diabetes. Free Radic Biol Med 63: 291-303, 2013.

32. Li Z, Dong X, Liu H, Chen X, Shi H, Fan Y, Hou D and Zhang X: Astaxanthin protects ARPE-19 cells from oxidative stress via upregulation of Nrf2-regulated phase II enzymes through activation of PI3K/Akt. Mol Vis 19: 1656-1666, 2013.

33. Kavitha K, Kowshik J, Kishore TK, Baba AB and Nagini S: Astaxanthin inhibits $N F-\kappa B$ and $W n t / \beta$-catenin signaling pathways via inactivation of Erk/MAPK and PI3K/Akt to induce intrinsic apoptosis in a hamster model of oral cancer. Biochim Biophys Acta 1830: 4433-4444, 2013.
34. Zhang XS, Zhang X, Wu Q, Li W, Zhang QR, Wang CX, Zhou XM, Li H, Shi JX and Zhou ML: Astaxanthin alleviates early brain injury following subarachnoid hemorrhage in rats: Possible involvement of Akt/bad signaling. Mar Drugs 12: 4291-4310, 2014.

35. Liu YW, Zhu X, Yang QQ, Lu Q, Wang JY, Li HP, Wei YQ, Yin JL and Yin XX: Suppression of methylglyoxal hyperactivity by mangiferin can prevent diabetes-associated cognitive decline in rats. Psychopharmacology (Berl) 228: 585-594, 2013.

36. Wu W, Wang X, Xiang Q, Meng X, Peng Y, Du N, Liu Z, Sun Q, Wang $C$ and Liu $X$ : Astaxanthin alleviates brain aging in rats by attenuating oxidative stress and increasing BDNF levels. Food Funct 5: 158-166, 2014.

37. Augusti PR, Conterato GM, Somacal S, Sobieski R, Quatrin A, Maurer L, Rocha MP, Denardin IT and Emanuelli T: Astaxanthin reduces oxidative stress, but not aortic damage in atherosclerotic rabbits. J Cardiovasc Pharmacol Ther 14: 314-322, 2009.

38. Choi SK, Park YS, Choi DK and Chang HI: Effects of astaxanthin on the production of NO and the expression of COX-2 and iNOS in LPS-stimulated BV2 microglial cells. J Microbiol Biotechnol 18: 1990-1996, 2008

39. Lee SJ, Bai SK Lee KS, Namkoong S, Na HJ, Ha KS, Han JA, Yim SV, Chang K, Kwon YG, et al: Astaxanthin inhibits nitric oxide production and inflammatory gene expression by suppressing I (kappa)B kinase-dependent NF-kappaB activation. Mol Cells 16: 97-105, 2003

40. Song X, Wang B, Lin S, Jing L, Mao C, Xu P, Lv C, Liu W and Zuo J: Astaxanthin inhibits apoptosis in alveolar epithelial cells type II in vivo and in vitro through the ROS-dependent mitochondrial signalling pathway. J Cell Mol Med 18: 2198-2212, 2014. 